\title{
AEROACOUSTIC RESEARCH IN EUROPE: THE CEAS-ASC REPORT ON 1999 HIGHLIGHTS
}

\author{
H. BODÉN ${ }^{1}$ AND S. SARIN ${ }^{2}$ \\ ${ }^{1}$ The Marcus Wallenberg Laboratory for Sound and Vibration Research, Department of \\ Vehicle Engineering, KTH, SE-10044 Stockholm, Sweden \\ ${ }^{2}$ SAAB AB, Future Products and Technology, SE-581 88 Linköping, Sweden
}

This paper is a report on the highlights of aeroacoustics research and development in Europe in 1999, compiled from information provided to the Aeroacoustics Specialists Committee (ASC) of the Confederation of European Aerospace Societies (CEAS). CEAS presently comprises the national Aerospace Societies of France (AAAF), Germany (DGLR), Italy (AIDAA), The Netherlands (NVvL), Spain (AIAE), Sweden (FTF), Switzerland (SVFW) and the United Kingdom (RaeS).

\section{INTRODUCTION}

The role of the CEAS-ASC is to serve and support the scientific and industrial aeroacoustics community in Europe. In this context "Aeroacoustics" includes all the aerospace acoustics and related areas. Each year the committee will highlight some of the research and development activities in Europe. This is the report on the 1999 highlights.

Contributions to this report has been made by the following people: R.J. Nijboer, E. Rademaker and J. B.H.M. Schulten (NLR), W. Dobrzynski (DLR), B.J. Tester (Rolls Royce plc), S. Lewy and J. Prieur (ONERA), H. Bodén (KTH), A. Hirschberg and S. W. Rienstra (TUE). 


\section{ENGINE NOISE}

\subsection{TURBOMACHINERY NOISE}

The EC funded project RESOUND (Reduction of Engine Source Noise through Understanding and Novel Design) addresses the challenge of reducing the noise at source, in particular turbomachinery noise, through (1) engine component aeroacoustic design and (2) through novel noise controlling devices that can be integrated within the engine structure.

Encouraging progress has been made towards developing and evaluating turbomachinery noise reduction technologies. The main achievement to date is the application of computational fluid dynamic codes to the design of lower noise turbomachinery components, fan rotors and low-pressure turbine exit guide vanes in particular. Furthermore, the effect of sound diffraction by the splitter of a turbofan engine was successfully modelled [1]. It was found that the effect of diffraction is larger on higher order radial modes than on lower order modes. Also diffraction effects are more important for noise coming from the engine section stator than for noise coming from the outlet guide vanes. (contributed byR.J. Nijboer and B.J. Tester)

Some significant improvement in the engineering models of fan buzz-saw noise and in the understanding of fan rotor-stator interaction tone noise and the benefits of swept/leant fan outlet guide vanes was obtained. The effect of the stator leading edge suction force on rotorstator interaction noise was found to be quite significant [2]. A representative advanced ducted fan design was used to study the effect on rotor-stator interaction noise. It appeared that the suction force is most effective when the generated modes have a low circumferential wave number m. (contributed by J.B.H.M Schulten and B.J. Tester)

An initial evaluation of the most promising active control devices supported by theoretical and experimental feasibility studies was made. One study concerned the effect of an upstream control grid consisting of a rotating ring of 11 cylindrical rods whose interaction with the rotor generates the same spinning modes as the rotor-outlet guide vanes assembly with the same levels, but out of phase. The control grid also generates other propagating spinning modes on harmonics of blade passing frequency (BPF). The measured spectra in Figure 1 show that $2 \mathrm{BPF}$ is $6 \mathrm{~dB}$ higher, and $3 \mathrm{BPF}$ is $15 \mathrm{~dB}$ higher with $\mathrm{ANC}$ on. However, BPF is the strongest tone as usual, and the overall sound pressure level is decreased by $6 \mathrm{~dB}$. (contributed by S. Lewy and B.J. Tester) 
Substantial progress was made towards the definition, design and manufacture of experimental hardware for verification testing of the down-selected low noise technologies and the resolution of test facility, rig and instrumentation problems. Fan noise tests on a datum fan rotor and stator and also combustion noise tests on a low emissions combustor was executed. (contributed by B.J. Tester)

\subsection{BASIC RESEARCH IN DUCT ACOUSTICS}

Within the Brite-Euram project DUCAT (basic research in duct acoustics and radiation) the development of a number of numerical models (FEM, BEM, coupled FEM/BEM, a nonlinear propagation model, a ray-acoustics model and a model for liners of extended reaction) have been completed. These models are partially complementary and partially overlapping, which offers the possibility to compare, verify, and choose the best modelling for each aspect of duct acoustics. The next steps will be the validation of the models by comparing with benchmark models and experimental results, and a comprehensive liner design exercise on a generic turbofan. (contributed by E. Rademaker)

An explicit multiple scales solution for modal sound propagation through slowly varying lined ducts with isentropic mean flow has been tested for aero-engine turbo fan inlet duct applications by comparison with Eversman's state-of-the-art numerical FEM solution [3]. Excellent agreement was found in both attenuation and iso-pressure contours, calculated for realistic conditions, inlet Mach number 0.5, dimensionless frequency $k a$ ranging from 10 to 50, circumferential mode numbers $m$ ranging from 10 to 40, wall impedance $Z=2-i$, first radial mode incident. See Figure 2. (contributed by S.W. Rienstra)

\section{HELICOPTER NOISE}

\subsection{CODE DEVELOPMENT}

The Kirchhoff code KIM has been applied to study the influence of the thrust on the noise radiation (in level and directivity) of a high-speed helicopter rotor with rectangular blades, starting from ONERA Euler calculations [4]. The aeroacoustic results confirm, at least for this type of blades and in the delocalised cases studied, that the thrust coefficient has a negligible effect on the radiated High-Speed Impulsive noise. (contributed by J. Prieur)

A code named CONGA able to predict blade vortex interaction noise using blade pressures measured by a small number of sensors has been validated on the basis of wind tunnel tests. The CONGA method is able to reproduce noise contours measured during flight, with a 
standard deviation lower than $3 \mathrm{~dB}$, starting from blade pressure data measured by only 8 sensors on a blade [5]. (contributed by J. Prieur)

\subsection{LOW-NOISE ROTOR OPTIMISATION}

Within a joint ONERA/DLR project (ERATO) a low-noise rotor optimisation methodology was globally verified through dedicated aeroacoustic wind tunnel tests [6]. Furthermore a high quality comprehensive data base including extensive blade pressure, strain gauge, microphone and wake measurements (at DNW using LLS and PIV), has been acquired which will serve for deeper understanding of physical phenomena in view of a future low noise rotor optimisation. (contributed by J. Prieur)

\section{AIRFRAME NOISE}

\subsection{FULL-SCALE HIGH-LIFT WING WIND TUNNEL EXPERIMENT}

As a worldwide first the DLR Institute of Design Aerodynamics has investigated the aeroacoustics of a full-scale Airbus A320 aircraft wing in the open test section of the German Dutch Wind Tunnel (DNW). This project on aircraft airframe noise sources - supported by the German Ministry of Education and Research and by DaimlerChrysler Aerospace Airbus aimed at understanding the dominant aeroacoustic source mechanisms of the wing high lift devices - namely leading edge slats and trailing edge flaps.

Farfield noise directivities were determined for different slat/flap settings, angles-of-attack and flow speeds, respectively. A planar-array of 100 microphones and a 3-m diameter acoustic mirror were used to localise and rank order different HLD component sources. The results revealed the dominance of certain additional noise sources related to construction details, which never were reproduced on scaled aircraft models. Slat noise and flapside-edge noise were found to be the dominating airframe noise component (see Figure 3). The research will continue with development of technically feasible noise reduction methods. (contributed by W. Dobrzynski)

\section{MISCELLANEOUS TOPICS IN AEROACOUSTICS}

In the BRITE-EURAM project FLODAC (Basic research in Flow Duct Acoustics) an aeroacoustical model for low frequency sound generation from a road tunnel ventilation fan (jet fan) has been developed. The acoustic model included the effect of the proximity to the tunnel roof and acoustic interaction between the two duct openings. A comparison between 
calculated and measured sound power from the fan showed excellent agreement [7]. The theoretical model developed has been used to give design rules on how to change the fan duct configuration to minimise the radiated sound power. (contributed by H. Bodén)

The FLODAC project also included studies of aeroacoustics of discontinuities in pipe systems such as bends, pipes bifurcations, wall perforations, diaphragms and diffusers [8-9]. (contributed by A. Hirschberg)

\section{REFERENCES}

1. R.J. Nijboer and P. Sijtsma 1999 Proceedings of the 6th International Congress on Sound and Vibration, Lyngby, Denmark. Sound diffraction by the splitter of a turbofan engine.

2. J.B.H.M. Schulten 1999 AIAA Paper 99-1951, Proceedings 5th AIAA/CEAS Aeroacoustics Conference, Seattle, WA. Unsteady Leading Edge Suction Effects on RotorStator Interaction Noise.

3. S. W. Rienstra and W. Eversman 1999 AIAA Paper 99-1821, Proceedings 5th AIAA/CEAS Aeroacoustics Conference, Seattle, WA. A numerical comparison between multiple-scales and FEM solution for sound propagation in lined flow ducts.

4. G. Rahier, and J. Zibi $199955^{\text {th }}$ Annual Forum of the American Helicopter Society, Montreal Canada, May 1999. A Fast Kirchhoff Integration Technique for Rotor Noise Directivity Predictions.

5. P. Spiegel 2000 to be presented at the $56^{\text {th }}$ Annual Forum of the American Helicopter Society, Virginia Beach, VA, May 2000. BVI Noise Prediction and Analysis Starting from Blade Pressures Measured on a Small Number of Points.

6. W. Splettstoesser, B. Van der Wall, B. Junker, K. Schultz, P. Beaumier, Y. Delrieux, P. Leconte and P. Crozier 1999 25th European Rotorcraft Forum, Rome, September 1999. Wind Tunnel Test Results and Proof of Design for an Aeroacoustically Optimised Rotor.

7. J.-G. Lalanne, H. Bodén, M. Åbom and R. Parchens 1999 Euromech Colloquium Cargèse Corsica 19-23 April 1999. Prediction of the sound power generated by a ducted axial fan.

8. N. B.Roozen, M. Bockholts, P. van Eck and A. Hirschberg 1998 Journal of the Acoustical Society of America, Vol 104 (1998) pp. 1914-1924. Vortex sound in bass-reflex ports of loudspeakers. Part I: Observation of response to harmonic excitation and remedial measures. Part II: A method to estimate the point of separation.

9. S. Dequand, S. Hulshoff, Y. Aurégan, J. Huijnen, L. van Lier and A. Hirschberg 1999 Proceedings of the 6th International Congress on Sound and Vibration, Lyngby, Denmark. Aero-acoustical response of a $90^{\circ}$ sharp bend. 


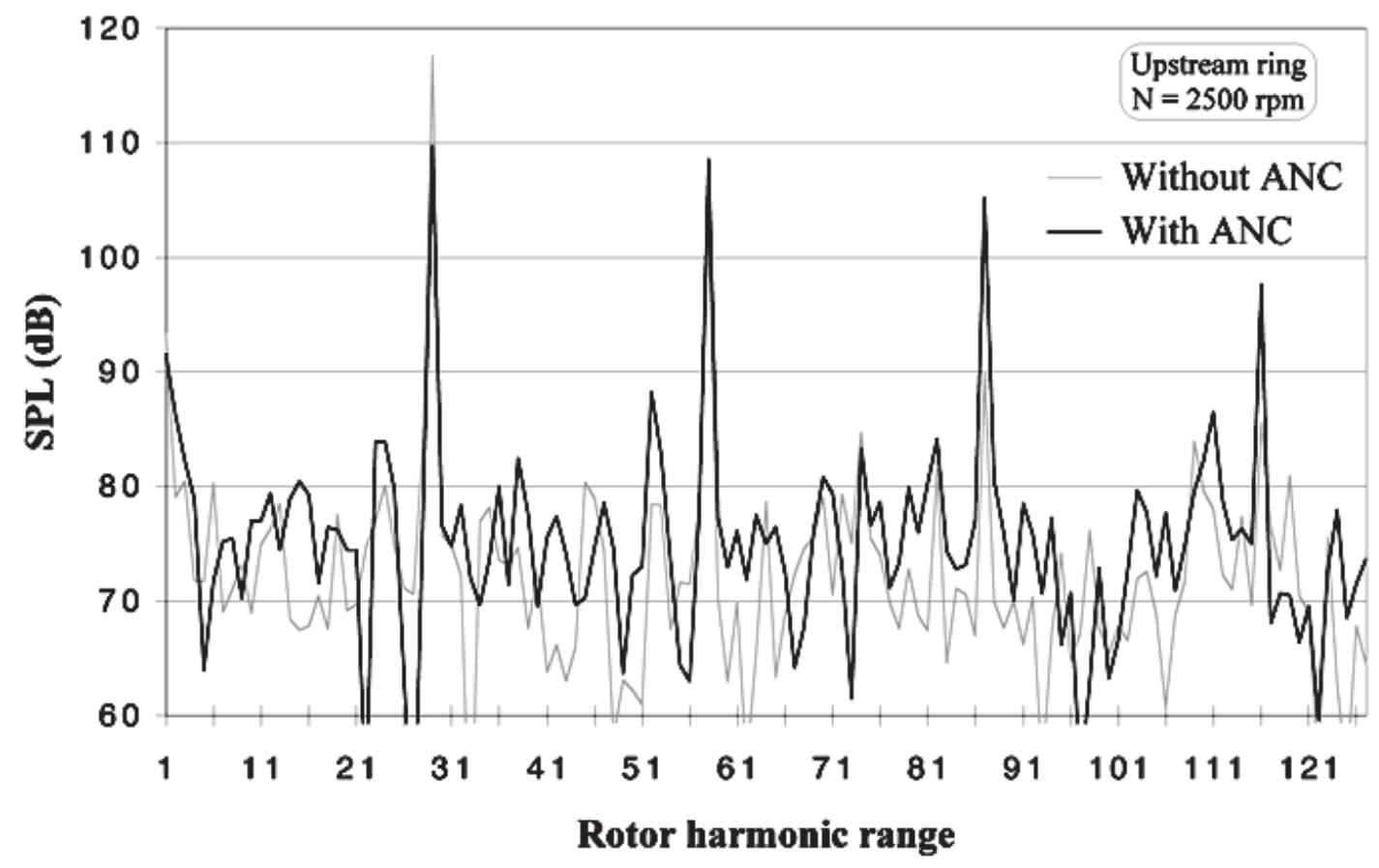

Figure 1. Measured spectra from test of new active control device for suppressing the blade passing tone due to interaction between rotor wakes and outlet guide vanes.
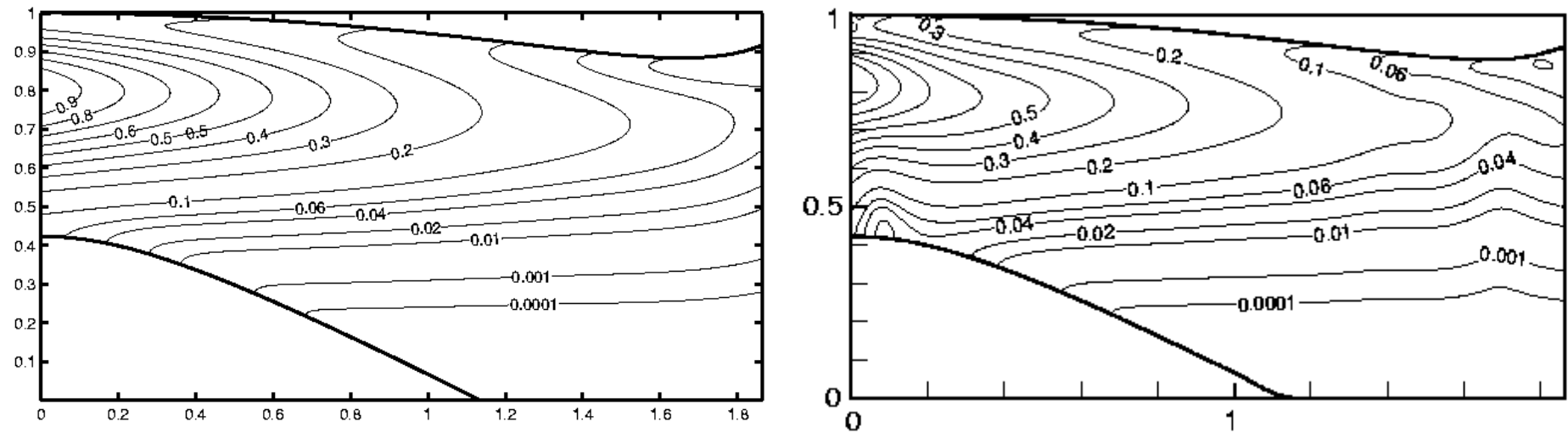

Figure 2. Iso-pressure contours from FEM (right) and multiple scales (left) solutions. 


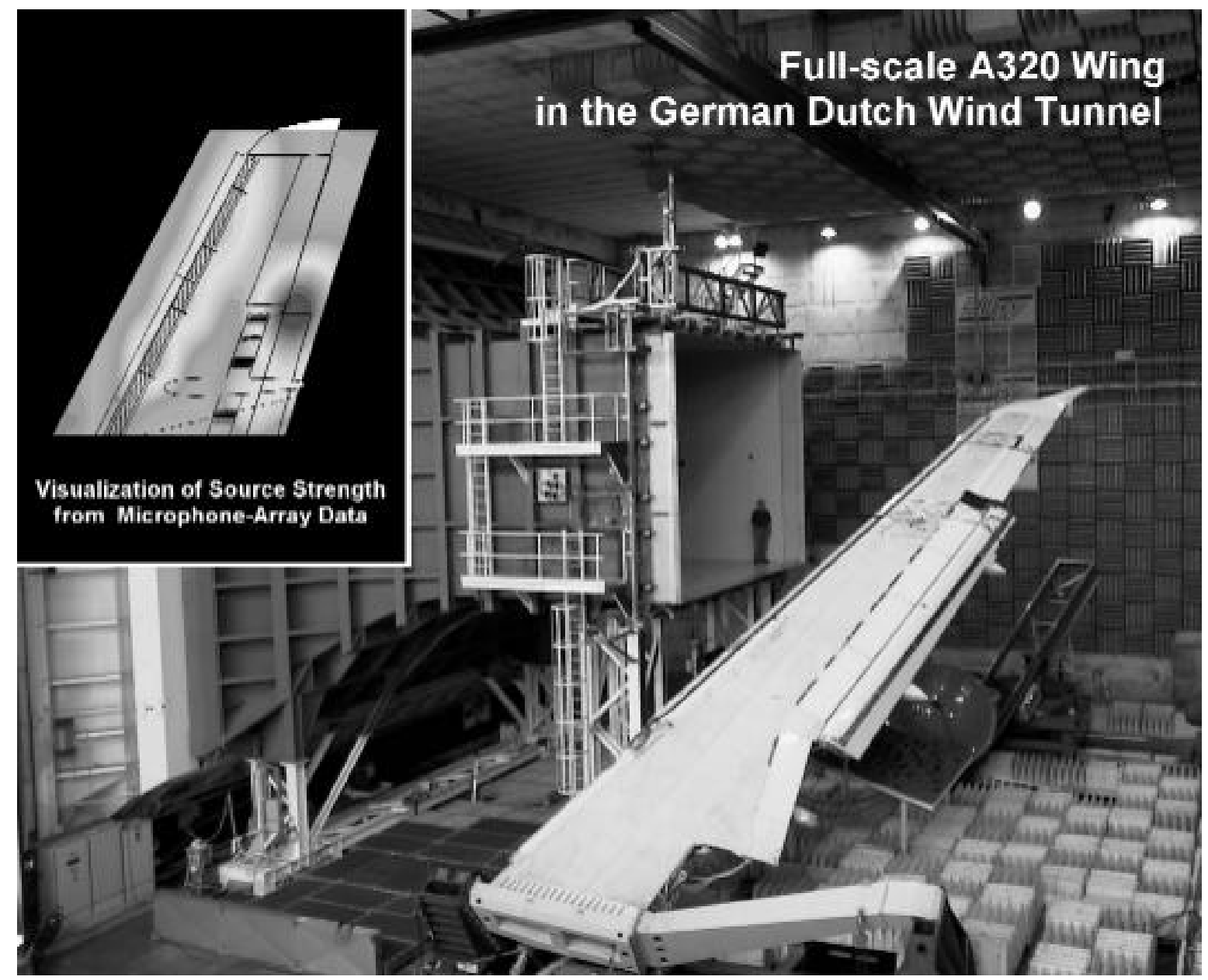

Figure 3. Photograph and source strength visualisation from full-scale test of an Airbus A320 wing in the German Dutch wind tunnel (DNW). 